ORIGINAL PAPER

\title{
ROBOTIC SURGERY IN THE ROMANIAN HEALTH SYSTEM
}

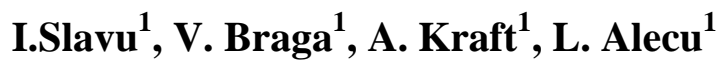 \\ ${ }^{1}$ The General Surgery Department, "Prof. Dr. Agrippa Ionescu" Emergency Clinical Hospital \\ Corresponding author: Lucian Alecu \\ Phone no. 0040212228129 \\ E-mail: lucianalecu@yahoo.com
}

\begin{abstract}
Robotic surgery appeared in the Romanian health system in 2008 with the purchase of the first two surgical robots. One of them belonged to "Dan Setlacec" Center of General Surgery and Liver Transplantation - Fundeni and the second to Floreasca Emergency Clinical Hospital. Funding was provided by the Ministry of Health special program. Since the first year of operation by the robotic system, the Fundeni Center has performed about 200 interventions. A key objective of the program was to identify the interventions that are cost efficient, i.e., around 9,000 RON for each surgical intervention. Subsequently, another 7 robotic systems were put into operation, out of which three are in urology and four in general surgery. Given the high cost of consumables and post-warranty maintenance, the operation of these robotic systems could only be done through the national health system, or private sector. The operation of the program allowed for an annual limited number of cases, and since 2013 the program funding has ceased. During this period, a significant experience was gained using robotic surgery in general surgery, urology and gynecology as well.
\end{abstract}

Keywords: Da Vinci, robotic surgery, health system

\section{Introduction}

The term robot was first used by Karel Kapek; it came from the Czech word robota that translates as forced labor [1]. Although nowadays robots are used to perform repetitive and dangerous tasks, important steps were taken to extend their usefulness spectrum. However, the passage of robotic systems in the medical field has been slow, but this period comes to an end especially regarding surgery. Robots that use telemedicine systems have been used to achieve the first transcontinental cholecystectomy [2]. The history of robotic surgery began with the system PUMA 560 which represented nothing more than an industrial robotic arm whose purpose was redesigned for biopsy brain tumors with high accuracy based on preoperative imaging [3]. Speculating this robotic arm skills, three years later Davies et al. performed the first transprostatic robotic resection, paving the way for a new therapy that will become the gold standard for the treatment of prostate cancer [4]. While the former concept of robotic surgery began to be used more often, it gave rise to the concept of telesurgery in the mid 1990s. It happened in the United States with significant investment from the military who wanted to obtain a robot that could operate on wounded soldiers through telesurgery on the front lines, so that the human operator is safely away. 


\section{Materials and methods}

One of the robotic systems that is still in use is the AESOP system developed by Computer Motion Inc., Santa Barbara, CA (California) which consists of an optical system used in laparoscopic surgery, that is controlled by the surgeon's voice. Other robotic systems include the Da Vinci robot developed by Intuitive Surgical Inc., Mountain View, CA and Zeus robotic system developed by Computer Motion Inc., Santa Barbara, CA. The last two systems are similar to each other in the way they operate. Both systems use the master-slave principle (i.e., they both depend on the operator and are unable to make decisions) and are equipped with multiple arms remotely controlled from a console by a surgeon. The Da Vinci system which has the most units in usage is composed basically of three main elements: a trolley that is equipped with an optical system, a master console and a trolley that is equipped with the arms and camcorder positioned at the operating table [5]. The master console is equipped with a computer that generates a 3-D image which can create a depth perception of the operative field. The instruments have 7 degrees of motion and can mimic the natural movements of the hand, unlike conventional laparoscopy which provides 5 degrees of movement [5].

\section{Why robotic surgery?}

Robotic surgery was developed to cover the deficits of laparoscopic surgery and to expand the indications of minimally-invasive surgery.

The advantages of robotic surgery:

- stability in the surgical instruments;

- mechanical advantages of the laparoscopic instruments which have a rotator tip of $360^{\circ}$ (Figure 2);

- better ergonomics for the operator;

- superior field view (3D);

- availability: minimally invasive approach available for cases where laparoscopy has no chance of success: for interventions that involve finesse gestures (e.g. dissection, fine sutures, reconstructions);

- low complication rate due to increased accuracy;
- robotics improve the ambidextrous assistance, improving their performance with the non-dominant hand.

\section{Discussions}

The advantages of robotic surgery for the surgeon include: greater dexterity, better view of the work field, greater accuracy. The physiological tremor of the surgeon is identified by the robot and removed through different filters.

The advantages of using robotic surgery for the patient include: reduced blood loss, reduced hospitalization and faster recovery, data confirmed by Ashutosh Tewari in a study published in November 2004. For example, in the case of a prostatectomy the average time for a patient to return to work varies from 49 days in the case of open surgery to 11 days in the case of robotic surgery [6].

Hospitals that own such a system will be automatically propelled to the forefront of surgery and development. However it should be noted that to be profitable the center which uses such a system needs to be a high-volume center according to Peter L. et al. who analyzed the profitability of such systems in the case of robotic prostatectomy. Its profitability increases with the number of cases operated. The Da Vinci Surgical System contributes to the economy of the hospital in three distinct ways: 1. Increases market share - the Da Vinci system can increase patient satisfaction, attract new patients, new references and new revenues. Also, the Da Vinci system may contribute to recruitment and retention of top talent and increases the surgical reputation of the hospital; 2. Saves money by decreasing hospitalization time: the Da Vinci system can immediately reduce hospitalization time to a larger number of patients who can now benefit from a minimally-invasive intervention; 3 . Streamlines operations: the Da Vinci system can immediately reduce the incidence of surgical complications, postoperative infections, blood transfusion, postoperative treatment such as painkillers and simplifies post-operative care. Performing a $1 \mathrm{~cm}$ incision the surgeon can make complex interventions using minimally invasive surgery and can reduce the 
postoperative infection rate to $0 \%$ in some cases [7].

We can also mention a low conversion rate, i.e., below $4 \%$, which proves the capabilities of robotic technology to provide surgeons with the possibility to conduct minimally invasive surgery in a secure, accurate and beneficial fashion to the patient [7]. According to El Hakim et al. using the precision, superior 3D viewing and minimally invasive approach, trauma and blood loss are significantly lower, by up to $80 \%$ than in open surgery and $50 \%$ in laparoscopy.

No specific complications are related to the surgical approach with the Da Vinci system. Complications are general and the same ones can occur during laparoscopic or classic surgery.

The disadvantages of robotic surgery Robotic surgery is still a new technology whose efficacy has not yet been proven. Another disadvantage is the cost, with a price tag of at least \$ 1 million per unit; for a large part of the healthcare systems of the world this technology is inaccessible. However, once the utilization increases, technology evolves and experience accumulates, costs will decrease in the future [8]. Another problem is the cost of improvement (upgrade) of these systems: as technology advances, the robotic system should be improved. A dilemma therefore arises as how much a hospital has to spend and how often has to meet these technological advances. Whatever the case, if such a system is to be cost-efficient, it should have broad, multidisciplinary applicability [9]. Lack of tools and compatible equipment is another drawback of this technology thus requiring additional personnel in the operating room to deal with this deficit. Currently, according to data published by Intuitive - the maker of the main system on the market, the Da Vinci robot, the world leader in number of systems installed and in operation is the United States of America with 2,295 systems installed, followed by Europe with 573 units, the majority being in France ( 88 units). Asia currently holds 383 robotic systems, South America 46 and the Middle East 36 units. As Fig. 3 shows, the number of robotic interventions had a steady growth from 2009 to 2014, a year which has seen a 9\% increase compared to 2013; in 2015 the number is expected to expand $11-13 \%$.

Due to the high cost of both acquisition and maintenance of these systems, the vast majority of operational programs are sponsored by governments. To date, there are over 25 such programs in 14 countries (Figure 1).

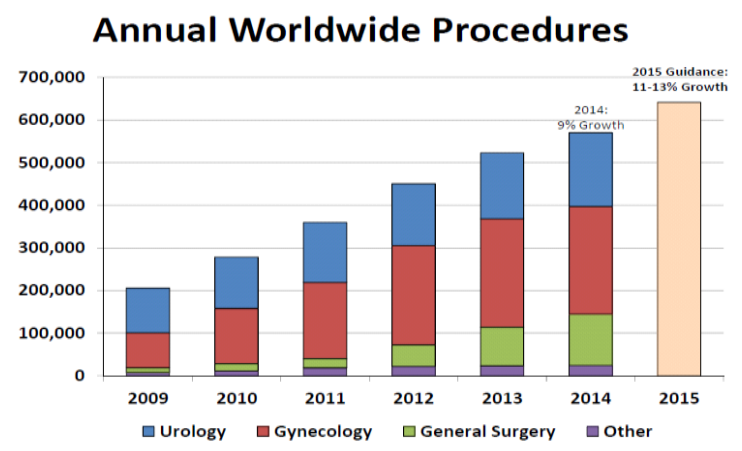

Figure 1 - The worldwide evolution of robotic surgery

\section{The Romanian experience}

At present, there are 7 robotic systems in Romania - five in Bucharest, one in Cluj and one in Timisioara (Figure 2).

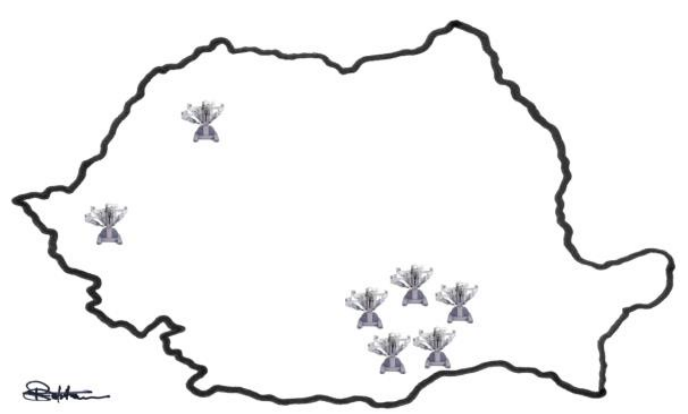

Figure 2 - The national distribution of robotic systems in Romania

The program started in 2008 by an Order of the Ministry of Health No. 417/431 via The National Program of Diagnosis and Treatment with the help of high performance technology which encapsulated the National Subprogram of surgical treatment of complex pathologies with the help of minimally invasive surgery, robotically assisted. Coordination was provided by "Dan Setlacec" Center of General Surgery and Liver Transplant, Fundeni Clinical Institute, Bucharest. The first two medical units that benefited from this program were Floreasca Emergency Clinical Hospital and Fundeni Clinical Institute. The sponsorship for the first year was for $120 /$ interventions; the amount 
allocated for each intervention was 8,000 RON, but by 2011 the number of sponsored interventions grew to 160 . In 2013, due to lack of funds, the whole program was stopped.

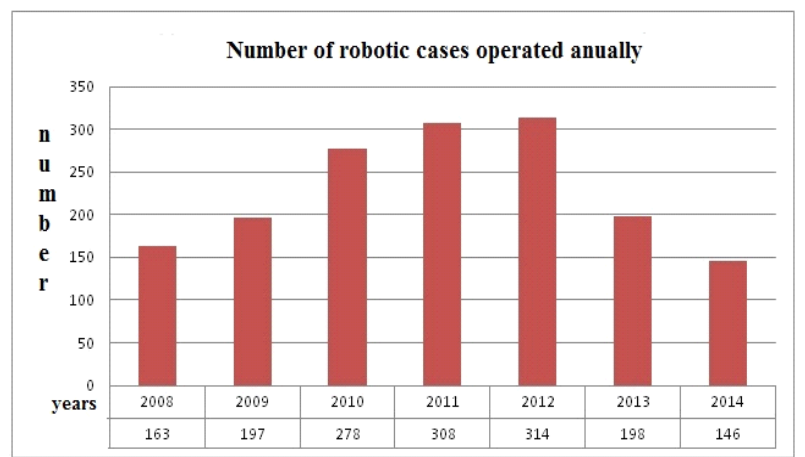

Figure 3 - Evolution of the number of cases operated robotically in Romania

After 1 year of robotic surgery, "Dan Setlacec" General Surgery Department of the Fundeni Clinical Institute published its first results which consisted of 153 interventions, five conversions, an average operative time of 171 minutes and 0 mortality.

As seen in the previous graph - Figure 3 - the number of robotic procedures in Romania increased steadily from the onset of the program in 2008 until 2012, when the program was left without sponsorship; thus, in only one year the number decreased from 314 to 198 interventions.

Regarding the surgical specialty where the robotic Da Vinci system was used, data show that general surgery used it the most often, unlike the international situation where gynecologic surgery and urology have the most interventions (Figure 4).

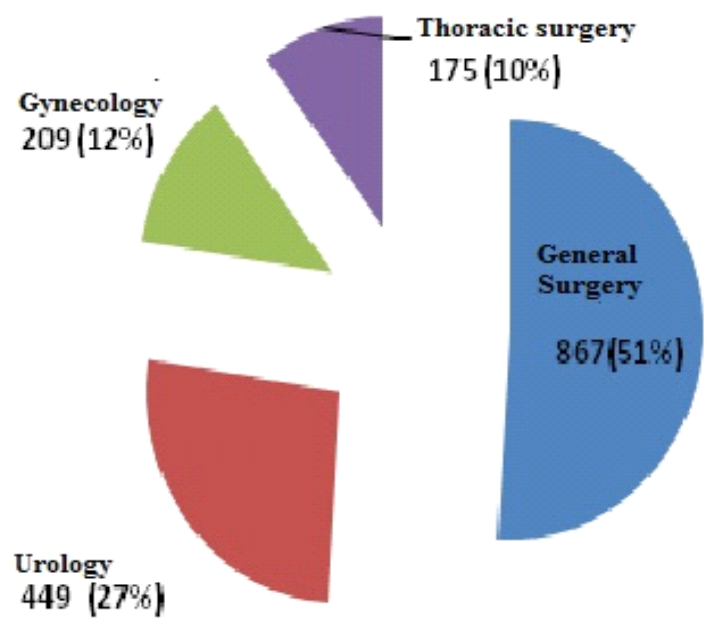

Figure 4 - Distribution by surgical specialties of the cases operated robotically in Romania
The Romanian experience in robotic surgery is reflected by more than 1,700 interventions performed nationwide, of which over 1,000 operations have been performed in Fundeni Institute of Clinical Surgery, "Dan Setlacec" General and Liver Transplant Center.

One of the segments of general surgery where the robotic system has proved its worth is colorectal surgery by the sphincter-saving procedures and total mesorectum excision (TME). The oncological results of robotic TME were analyzed by Alberto Patriti et al. (2009) who compared these results with those obtained from conventional laparoscopy and demonstrated that robotic TME can have a shorter operative time and a lower conversion rate. Both distal and circumferential margins were free of tumor and the number of lymph nodes harvested was similar for both methods. So it can be concluded that robotic TME meets the criteria for cancer cure, increases the ease of surgical maneuvers (dissection) simultaneously decreasing operative time [10].

Due to the high cost of these systems, in Romania's private sector there are no such systems. There is one, but it functions within a public-private mechanism.

One way for robotic surgery to develop is by research grants. At the moment there are two such systems that function and both were purchased with the help of European research grants.

\section{Conclusions}

1. Although it has experienced a difficult road, robotic surgery in Romania has acquired considerable experience of over 1,700 cases operated.

2. The main obstacle to the development of robotic surgery was the discontinuity of funding from the public health system.

3. Currently, the development of robotic surgery in the private sector is limited - even absent, but given that this segment is developing every year the hopes are that in the future such services will be provided.

4. Acquisition of robotic systems by European research programs is one of the ways to develop this therapy. 


\section{References}

[1]Satava RM. Surgical robotics: the early chronicles: a personal historical perspective. Surg Laparosc Endosc Percutan Tech. 2002;12:6-16. [PubMed]

[2]Cheah WK, Lee B, Lenzi JE, et al. Telesurgical laparoscopic cholecystectomy between two countries.

[3]Surg Endosc. 2000;14:1085. Kwoh YS, Hou J, Jonckheere EA, et al. A robot with improved absolute positioning accuracy for CT guided stereotactic brain surgery. IEEE Trans Biomed Eng. 1988;35:153-161.

[4]Davies B. A review of robotics in surgery. Proc Inst Mech Eng. 2000;214:129-140

5. Kim VB, Chapman WH, Albrecht RJ, et al. Early experience with telemanipulative robot-assisted laparoscopic cholecystectomy using Da Vinci. Surg Laparosc Endosc Percutan Tech. 2002;12:34-40.

[6]Yim GW, Jung YW, Paek J, Lee SH, Kwon HY, Nam EJ, Kim S, Kim JH, Kim YT, Kim SW, Transumbilical single-port access versus conventional total laparoscopic hysterectomy: surgical outcomes. Am J Obstet Gynecol. 2010 Jul; 203(1):26.e1-6.

[7]Payne TN, Dauterive F. A comparison of total laparoscopic hysterectomy to robotically assisted hysterectomy: surgical outcomes in a community practice. J Minim Invasive Gynecol. 2008 May-Jun; 15(3): 286-91.

[8]Institute for Clinical and Economic Review (ICER). Final Appraisal Document. Active Surveillance \& Radical Prostatectomy for the Management Of Low-Risk, Clinically-Localized Prostate Cancer. September 11, 2009.

[9]Escobar PF, Starks D, Fader AN, Catenacci M, Falcone T Fertil Steril. Laparo endoscopic singlesite and natural orifice surgery in gynecology. 2010 Dec; 94(7):2497-502.

[10]Patriti A, Ceccarelli G, Bartoli A, Spaziani A, Biancafarina A, Casciola L. Short- and mediumterm outcome of robot-assisted and traditional laparoscopic rectal resection. JSLS 2009; 13: 176183. 